\section{The Meaning of Existence.}

What Sir James Jeans says (Nature, Feb. 28, p. 304 ) is, of course, quite true ; but it scarcely answers Prof. Muirhead's question. May I try to explain what existence means in physics?

Primarily, it means the truth of some law having the form which defines a substance or object. To say that silver exists is simply to declare that it is possible to demonstrate experimentally the association of the 'properties of silver', a certain density, melting point, and so on. No serious person during the last fifty years has believed that the ether exists in this sense.

Secondarily, it means the truth of some theory. A theory is not true unless $(a)$ true laws can be deduced from it and $(b)$ its hypothesis is intrinsically plausible. This intrinsic plausibility is often derived from analogy with laws ; if the laws from which it is derived are of the aforesaid form, the theory implies the existence of something. Kelvin held that the ether exists because he believed in a theory the hypothesis of which had an intrinsic plausibility arising from an analogy with the laws describing the oscillations of a continuous medium. That theory is now known to be false on ground $(a)$; Kelvin's ether, therefore, does not exist. But another theory has been put forward the hypothesis of which is a set of equations having an imperfect analogy with those describing the geometrical laws of space-which might be occupied by a continuous medium. This theory is certainly true on ground $(a)$. Some of us (including Sir James Jeans, I think) do not feel that the theory derives any intrinsic plausibility from this loose and indirect connexion with a continuous medium; we therefore reject the existence of the ether on ground $(b)$. Others feel that it does ; the idea of a continuous medium filling all space helps them to understand and believe relativity ; for them both $(a)$ and $(b)$ are satisfied, and they hold that the ether exists. Since the difference is one of personal taste (not, as Eddington suggests, of words), there is no way of deciding between us.

Two comments may be added. (1) Physicists do not apply these criteria of existence consciously ; they can be brought to light only by examining what physicists do believe to exist. That is inevitable; for physies does not arrive at truth by formal reasoning based on fixed principles; it employs reasoning only to expound and to co-ordinate conclusions reached by some more instinctive process. (2) Existence in the physical sense has, of course, no meaning outside the region of experience that can be analysed into laws; and it is hopeless to explain what physical existence means to anyone to whom a law is not a primary and unanalysable conception. NORMAN R. CAMPBELL.

155 Hagden Lane,

Watford, Herts, Mar. 1.

I HAVE to thank Sir James Jeans for his courteous reception (NATURE, Feb. 28, p. 304) of my recent communication in these columns. I had no intention of starting metaphysical hares in this field, but merely to express my agreement with him in holding that physical discussions are also apt to have their hares unless we begin with definitions of our terms. His very interesting letter brings this home from the side of the contrast between the physicist of forty years ago with his assumption of "a vast independent universe of concrete machinery, unthinkingly and unconsciously accepted as his universe of discourse", and the scientific worker of to-day, who finds his universe of discourse in "a phenomenal universe as apprehended by his brain ". Though I should perhaps use other terms for the latter, I think that thís well describes the change in the intellectual atmosphere brought about by recent developments in physics, to which I began by alluding in my former letter. The "vast independent universe of concrete machinery" had not only established itself in the mind of the physicist of those days as independent, but also it was endangering the independence of all other universes by reducing them to appanages of itself, as the only reality, containing the " promise and potency " of all other forms of existence.

Since then, not only have other universes (for example, those of mind and its creations in art, law and morality, science itself, to mention no others) staked out claims of their own in a lateral direction, but also physies itself, in extending its work vertically, has struck on a world to which the old conception of "concrete machinery" seems to be no longer applicable and the method of behaviour of which has to be expressed in quite other terms. How this world is to be co-ordinated with that of conerete machinery is, I suppose, one of the questions that at present occupy and are likely long to occupy the foremost scientific workers.

What Sir James Jeans, among others, as I understand him, has suggested, is the paradoxical conclusion that, in thus deepening the view of its own universe, physics has revealed analogies between it and other universes that prima facie belong to an altogether different order, and has thus opened up the possibility of a synthesis founded, not on their subordination to mechanical conceptions, but on the free right of each to develop its own underlying assumptions in a republic of inter-related universes.

If there is any truth in this view, we may thank discussions like that out of which this correspondence rose for bringing it more clearly to light. Its precise bearing on the particular point at issue is another matter. My own amateurish suggestion was not exactly that which Sir James Jeans attributes to me. I mentioned the world of values as a conspicuous example of a universe of discourse beyond the field of "pointer readings"; but "values" have enough to answer for without being loaded with the problem of ether. Ether might be shown to serve a purpose, to have, if we like. explanatory value, and that, I suppose, is what its advocates try to show; but on that I am not competent to express an opinion.

If I may restate it in other terms, perhaps less ambiguous than those I used, what I meant to suggest was, first, that whatever can be said for the existence of such an entity, it seems to be becoming more and more obvious that it does not fall within the universe defined as "that which is continuous with the felt, waking body"; and secondly, that the insistence upon it nevertheless as a real entity may not be unconnected with what Meyerson calls the " natural metaphysic" of the human mind which prompts it to seek for substantiality in what it takes as "given". For, in spite of what writers like Whitehead are saying in criticism of the old category of substance, we may drive it out with a fork but it will always recur; and if there is no place for it in the universe of mathematical physics, there will always be those who will try to find one elsewhere.

\section{J. H. Muirhead.}

\section{The Use of Govers on Lambs in Biological} Work on Wool.

MY fellow-workers on wool in the northern hemisphere will soon be welcoming a crop of lambs. I should like to tell them of the usefulness of the simple device of light covers. Covers for older sheep have already proved most valuable in work on the chemistry of yolk by my colleague, Mr. W. G. Sutton. The

No. 3204, VoL. 127] 